\title{
Isabelle Stengers, Une autre science est possible ! Manifeste pour un ralentissement des sciences
}

Paris, Les empêcheurs de penser en rond - La découverte, 2013

\section{Grégory Dufaud}

\section{(2) OpenEdition}

\section{Journals}

\section{Édition électronique}

URL : https://journals.openedition.org/artefact/7240

DOI : $10.4000 /$ artefact.7240

ISSN : 2606-9245

\section{Éditeur :}

Association Artefact. Techniques histoire et sciences humaines, Presses universitaires du Midi

Édition imprimée

Date de publication : 18 juin 2015

Pagination : 217-219

ISBN : 978-2-271-08155-1

ISSN : 2273-0753

\section{Référence électronique}

Grégory Dufaud, «Isabelle Stengers, Une autre science est possible ! Manifeste pour un ralentissement des sciences », Artefact [En ligne], HS 01 | 2015, mis en ligne le 27 avril 2021, consulté le 05 mai 2021. URL : http://journals.openedition.org/artefact/7240 ; DOI : https://doi.org/10.4000/artefact.7240

Ce document a été généré automatiquement le 5 mai 2021.

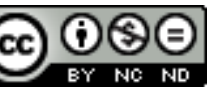

Artefact, Techniques, histoire et sciences humaines est mise à disposition selon les termes de la Licence Creative Commons Attribution - Pas d'Utilisation Commerciale - Pas de Modification 4.0 International. 


\section{Isabelle Stengers, Une autre science est possible! Manifeste pour un ralentissement des sciences}

Paris, Les empêcheurs de penser en rond - La découverte, 2013

\section{Grégory Dufaud}

\section{RÉFÉRENCE}

Isabelle Stengers, Une autre science est possible ! Manifeste pour un ralentissement des sciences, Paris, Les empêcheurs de penser en rond - La découverte, 2013, 200 p.

1 Cet ouvrage est un recueil de textes, déjà publiés ou non, de la philosophe Isabelle Stengers qui souhaite bousculer les routines scientifiques en appelant à un ralentissement des sciences. Qu'est-ce que cela signifie ? Comment le mettre en œuvre ? Dans quel but ? C'est ce qu'elle s'emploie à expliquer par touches successives, avec précision et clarté.

2 Dans le premier chapitre, elle pose la nécessité de ce qu'elle nomme une " intelligence publique des sciences " par le biais de la mobilisation de citoyens éclairés qui jugeraient la production des savoirs scientifiques par leur mise à l'épreuve, non pas depuis l'intérieur, mais depuis l'extérieur du milieu scientifique. L'intelligence publique des sciences aspire à actualiser, en l'approfondissant, le lien entre culture et politique. L'intelligence publique des sciences est en retour susceptible d'affermir l'autorité des scientifiques à la condition essentielle que les scientifiques cessent de mettre à distance l'opinion et le public au motif de leur autonomie et du caractère singulier de leur activité. Autrement dit, les scientifiques doivent accepter les objections et les remarques faites par les connaisseurs. Pour I. Stengers, l'intelligence publique des sciences apparaît alors comme un moyen de protéger les scientifiques contre l'entreprise de discrédit menée par ceux que Naomi Oreskes et Erik M. Conway ont appelé les « marchands de doute $»^{1}$. 
3 La question de l'intelligence publique des sciences pose le problème de l'étoffe du chercheur qu'interroge le deuxième chapitre. En recourant au terme d'« étoffe ", I. Stengers évoque " la qualification d'une différence liée à des questions qui se posent mais ne seront pas posées, à une manière de serrer les dents et de résister à ce qui dès lors devient une tentation " (p. 30). L'étoffe renvoie alors à une distinction vécue sur le mode de l'évidence. Le vrai chercheur, celui qui a l'étoffe donc, se caractérise pour I. Stengers par sa méfiance à l'endroit de l'opinion, jugée subjective et irrationnelle. C'est cette étoffe qui l'empêche de s'interroger sur la portée de ses recherches, car le faire consiste à repenser sa relation avec le public. C'est toujours l'étoffe qui conduit les scientifiques à disqualifier les questions qu'ils considèrent comme non légitimes. Contre les réactions de mobilisation disciplinaire, I. Stengers appelle à la " possibilité d'une démobilisation ». Elle entend par là l'éventualité que les chercheurs se posent des questions qui importent au-delà de leur domaine de recherche et de leur discipline. D'après elle, une manière d'y parvenir est de développer des dispositifs associant chercheurs et citoyens, dispositifs qui signalent l'ignorance générée à l'occasion de la production de savoirs nouveaux et éprouvent la fiabilité des propositions scientifiques.

4 La démobilisation doit s'accompagner d'un ralentissement de la science dont le troisième chapitre esquisse les modalités. Selon I. Stengers, ce ralentissement est la condition nécessaire à la création de « contrastes »-terme repris de Bruno Latour au sein du régime de véridiction scientifique, dominé par le modèle des sciences expérimentales, comme en témoignent les pratiques d'évaluation actuellement en vigueur, à commencer par le système des referees ou pairs compétents. Ce ralentissement pose non seulement la question de la formation des scientifiques et des pratiques de jugement qui sont les leurs, mais il suppose aussi une culture de la symbiose, c'est-à-dire " la capacité de chaque protagoniste à se présenter avec ce qui lui importe et à savoir que ce qu'il apprendra de l'autre devra toujours être compris comme réponses aux questions qui, pour lui, importent » (p.80). Au nom de ce qui nous importe vraiment, le ralentissement invite donc à un effort réflexif de la part des scientifiques et à la création de rapports nouveaux entre tous les acteurs de la science.

$\mathrm{Du}$ ralentissement, I. Stengers passe à la lenteur dans le quatrième chapitre où elle développe " un plaidoyer pour une science "slow" ». À travers l'apologie de la lenteur, deux caractéristiques de la science sont pointées : la première concerne le caractère soi-disant désintéressé de la science, tandis que la seconde renvoie au caractère expurgé des lieux de production des savoirs. Le développement d'une science lente ne consisterait plus pour les scientifiques à accumuler les faits et à laisser les acteurs économiques et étatiques décider de ce qu'il faut en faire. Au contraire, la science lente milite pour cette intelligence publique des problèmes évoquée au premier chapitre de l'ouvrage, laquelle suppose la création d'une forme nouvelle de sociabilité destinée à éprouver la fiabilité des propositions scientifiques. Pour les situations de controverse, I. Stengers propose ainsi la mise en place de « consultations » qui auraient la faculté, le " pouvoir ", nous dit-elle, de faire penser ensemble des participants ébranlés dans leur schème de pensée.

6 L'abandon par les scientifiques de leur condescendance à l'égard du public et de tout ce qui ne relève pas de leur sphère d'activité est précisément, comme I. Stengers l'explique dans le cinquième chapitre, ce qui peut permettre de " civiliser » la science : celle-ci ne se définirait plus par sa position d'autorité, mais par la pertinence de ses propositions. L'invitation à civiliser la science renvoie à un projet d'écologie politique 
qui considère que les questions jugées non scientifiques sont d'égale valeur que celles dites scientifiques. Cette écologie politique suppose trois éléments qui ont été évoqués au fil du livre : la « mise en politique des sciences », le refus d'une économie de la connaissance marquée par la logique prédatrice du capitalisme et la réforme de la formation scientifique. Néanmoins, I. Stengers pointe une limite qui tient à la scène politique et aux procédés à inventer : "On pourrait dire que l'écologie politique demande une opération de mise à égalité de ceux qui acceptent de se réunir autour d'une question en respectant la contrainte politique » (p. 137).

7 La réflexion d'I. Stengers est donc précieuse en ce qu'elle invite à reconnaître la pluralité de la science, à s'interroger sur l'économie actuelle des connaissances et à repenser la place de la science dans nos sociétés contemporaines. L'ouvrage s'achève alors par un court texte de William James de 1903, dans lequel celui-ci se penche sur les effets du doctorat sur l'enseignement et la recherche. Le texte est longuement présenté par Thierry Drumm qui en précise les enjeux en les situant dans le cadre de la pensée jamésienne et du pragmatisme. Le point de départ du texte de James est l'« incident " survenu à l'un de ses anciens étudiants qui, recruté à un poste d'enseignant à Bryn Mawr College, voit son emploi menacé lorsque la direction apprend qu'il n'est pas docteur. Pour obtenir le " bibelot académique ", il s'engage dans l'urgence à rédiger sa thèse qu'il finit par soutenir, "lav[ant] la tache qui salissait sa réputation et rétabli[ssan]t son établissement dans des relations convenables dans le monde ». Pour James, l'épisode témoigne de ce qu'est devenu le monde universitaire de son temps, où " le snobisme académique " le dispute au conformisme. Le doctorat fonctionne ainsi comme un poulpe qui prive les individus de leur capacité à penser et s'exprimer. Pour James, la mainmise du « poulpe du doctorat » représente une menace sérieuse. Aussi, pour la contrer, il propose que le doctorat soit octroyé à tous ceux ayant consacré du temps sur un sujet et demande à ce que les universités, à l'occasion des recrutements, prêtent davantage attention à la " substance ». James était attentif aux finalités que se donnent les institutions universitaires et les personnes y appartenant : c'est bien de cela dont traite aussi I. Stengers.

\section{NOTES}

1. Naomi Oreskes, Erik M. Conway, Les marchands de doute, Paris, Le Pommier, 2012. 


\section{AUTEURS}

GRÉGORY DUFAUD

Centre d'études des mondes russe, caucasien et centre-européen (CERCEC) 